\title{
Therapeutic Immunomodulation with Mesenchymal Stromal Cells: The Need for In Vivo Clues
}

\author{
L. Dolcetti and F. Dazzi \\ Department of Haematological Medicine, King's College London School \\ of Medicine, London, United Kingdom \\ Corresponding Author: francesco.dazzi@ kcl.ac.uk
}

Received 15 January 2015; Accepted 12 May 2015;

Publication 12 May 2015

\begin{abstract}
Mesenchymal stem/stromal cells are effective therapeutic agents for a variety of pathological conditions. However, the molecular mechanisms underlying their action remain largely unknown and biased by in vitro studies. In this concise review we have described recent advances in MSC therapeutics based on in vivo observations. We have also discussed the possibility of using engineering approaches to improve and facilitate deciphering MSC functions.
\end{abstract}

Keywords: Mesenchymal stem/stromal cell, immunomodulation, immunosuppression, tolerance, scaffold, regenerative medicine.

\section{Introduction}

The first appearance of mesenchymal stem cells (MSC) in the literature dates back to the early '70s in a work conducted by Friedenstein, in which MSC were isolated from rodent bone marrow as a plastic adherent fibroblast-like cell population capable of in vitro clonogenic expansion and condrogenic, osteogenic and adipogenic differentiation $[1,2]$. The descriptive nomenclature that is currently used has been originally conceived by Caplan [3], who

International Journal of Translational Science, Vol. 1, $23-40$.

doi: 10.13052/ijts2246-8765.2015.003

(C) 2015 River Publishers. All rights reserved. 
included other characteristics that contributed to greatly increase the interest in this cell type, such as the suitability for tissue engineering practice and the capability to secrete a broad spectrum of bioactive molecules. It is not surprising that MSC are being tested in many clinical trials for ailments of different aetiology, organ distribution and underlying pathogenesis [4]. The preparation of clinical-grade MSC involves a phase of isolation and ex vivo expansion that enrich an originally rare population until a 'therapeutic' dose is achieved [5]. Despite their convincing therapeutic activity, it is not yet known to what extent the medicinal reagent resembles the primary cell population sourced from the tissue [6]. Therefore, a better understanding of the differences between native and cultured MSC [7] could shed light on their biology and the pathways involved in producing clinical benefits.

Culture expanded MSC represent a heterogeneous cell population, ranging from mesenchymal progenitors, stromal fibroblasts and pericytes. Whilst not expressing markers that typically pertain to hematopoietic and endothelial lineage, a set of stromal markers such as CD90, CD105, CD73, CD106, CD166, CD44 and Stro-1 are frequently detected [8], although with some variation related to the tissue from which they are isolated [9]. Initially sourced from adult bone marrow (BM-MSC), MSC can be successfully expanded from adipose tissue (AD-MSC), as well as from other anatomical districts such as lung, heart, hair follicles (HFSC), granulosa (GC), dental pulp [10]. There is now much interest in MSC derived from tissues of foetal or neonatal provenance: umbilical cord (UC-MSC) and cord blood (CB-MSC) [11], umbilical cord Wharton's jelly (WJ-MSC), placenta decidua basalis (PDB-MSC) and amniotic membrane (AM-MSC) [12] because of the ease of procurement and the advantageous logistics of fast cell expansion in vitro [11]. However, there is no convincing evidence in support of choosing one source rather than another for therapeutic purposes. The option of adult MSC could be justified by the necessity of using an autologous product which would not be subject to immunological rejection. The major limitations to such an approach are an inefficient cell expansion from adult donors and evidence that MSC derived from some patients appear to be therapeutically less efficacious than those from healthy donors [13].

MSC are clinically interesting for their regenerative properties and they found ample applications regarding liver [14], cardiovascular [15], musculoskeletal and orthopaedic [16] (including cartilages defects following trauma [17], osteoarthritis [18], rheumatoid arthritis [19], osteoporosis, osteonecrosis and osteogenesis imperfecta [20], degenerative disc disease [21]), 
neurological (including amyotrophic lateral sclerosis (SLA) [22,23] and Alzheimer's disease [24]), renal and autoimmune diseases [25, 26].

Those related to immune-mediated disorders are probably the most convincing clinical applications of MSC because of their immunomodulatory properties. A number of successful clinical trials have been conducted in severe acute GvHD [27], solid organ transplantation [28] and in autoimmune disorders like Crohn's disease [29] and multiple sclerosis [30].

\section{MSC Immunosuppressive Properties In Vivo}

MSC immunosuppressive activity has largely been described both in vitro and in vivo, but information on mechanistic details in vivo are still limited. This is particularly important in light of the observation that MSC are not constitutively immunosuppressive but need a 'licensing' process usually provided by inflammatory molecules [31]. Among others, IFN- $\gamma$ seems to be one of the most potent licensing factors [32], while TNF- $\alpha$ and IL-1 synergise with IFN- $\gamma$ priming [33]. Immunosuppressive licensing is part of the more general concept of MSC polarization toward an inhibitory rather than a pro-inflammatory phenotype, as a consequence of the specific duration and concentration of a stimulus [34] as well as the complex of interactions with cells of both innate and adaptive immune systems [35, 36]. By way of example 'unlicensed' MSC can promote adverse effects instead of being therapeutic, as demonstrated by the accelerated graft rejection in rats receiving MSC 4 days before heart transplant [37], and in certain condition, MSC immunosuppressive functions can be inhibited instead of licensed by soluble factors released by pre-activated lymphocytes [38].

One of the crucial modalities by which MSC exert their immunosuppressive properties is through essential amino acid-metabolizing enzymes such as indoleamine 2,3-dioxygenase (IDO) and nitric oxide synthase, inducible (iNOS), cyclooxygenases (COX) metabolite prostaglandin E2 (PGE2), tumor necrosis factor $\alpha$-induced protein 6 (TSG6) and transforming growth factor $\beta$ (TGF $\beta$ ). MSC can also elicit other immune-regulatory cell populations such as regulatory T-cells (Treg), myeloid derived suppressor cells (MDSC) and tolerogenic dendritic cells (DC).

Despite the types of mechanism are somehow similar in nature, there are species-specific differences. For example, MSC sourced from monkey, pig and human primarily employ IDO as effector molecule whilst MSC from mouse, rat, rabbit and hamster utilize iNOS $[39,40]$. This discrepancy has been already described in a variety of models including also antimicrobial effector 
function [41] or in other cell types such as macrophages [42]. However, human skin-derived MSC seem able to produce NO especially when analysed in subject with psoriasis [43].

IDO acts on tryptophan to produce $\mathrm{N}$-formyl-kynurenine. Tryptophan depletion and the accumulation of kynurenine pathway metabolites contribute to modulate the activity of the immune, reproductive and central nervous systems [44]. In vitro exposure of MSC to IFN- $\gamma$ up-regulates IDO expression whilst TNF- $\alpha$ by itself does not but can synergise with IFN- $\gamma$ [45]. In vivo IDO up-regulation, along with other immunoregulatory factors, has been described in a pig model of femoral artery transplantation following the infusion of autologous MSC. However, since IDO was detected on the vascular graft this study did not unambiguously determine if IDO up-regulation occurred within MSC or in other cell types [46]. MSC have been proved to be directly responsible for the increase of kynurenine levels, in a model of kidney allograft tolerance. IDO ${ }^{-/-}$MSC or the IDO inhibitor 1-methyl-tryptophane abolished the kynurenine increment and consequently allograft tolerance. This work also reported an increase in the percentage of $\mathrm{CD} 4^{+} \mathrm{CD} 25^{+} \mathrm{Foxp} 3^{+}$ Treg and the failure to achieve graft tolerance upon antibody-induced CD25 ${ }^{+}$ depletion, indicating the presence of further contributing mechanisms in the induction of MSC mediated immunosuppression [47]. Studies in humans have corroborated the primary role of IDO up-regulation. Wang and colleagues demonstrated that the intravenous infusion of UC-MSC, into 6 patients affected by systemic lupus erythematosus produced an increased level of serum kynurenine. However, no changes in serum tryptophan concentrations were detected. The increase in circulating $\mathrm{CD} 8^{+} \mathrm{T}$-cells number and serum level of IFN- $\gamma$ have been suggested to induce IDO expression in MSC [48]. Interestingly, as previously reported, patient derived MSC were unable to up-regulate IDO upon in vitro IFN- $\gamma$ treatment.

MSC-related iNOS activity has been proved to contribute to immunosuppression in rodents. Adult rat derived MSC were effective in improving recipient survival after receiving allograft heart transplantation. MSC treatment completely lost the beneficial effect upon administration of iNOS inhibitor aminoguanidine [49]. Similarly, in a model of collagen-induced arthritis (CIA), iNOS ${ }^{-/-}$MSC were less effective in comparison to wild-type MSCs in reducing clinical severity [50].

iNOS expression in vivo could be negatively regulated by the suppressor of cytokine signalling 1 (SOCS1) and p53. As recently demonstrated, MSC shRNA silenced for SOCS1 have been shown to dampen OVA induced delayed-type hypersensitivity (DTH), as well as to boost the growth of 
B16-F0 melanoma thus suggesting an increased immunosuppressive capability. [51]. The co-injection of $\mathrm{p} 53^{-/-}$MSC with a B16-F0 melanoma increased tumor growth in an iNOS dependant manner, [52]. Both studies indicated the co-existence, within the IFN- $\gamma / \mathrm{TNF}-\alpha$ activating axis, of negative regulatory checkpoints that could promote the fine tuning of in vivo MSC immunosuppressive activity.

Prostaglandins have a variety of physiological and immunological effects [53, 54]. PGE2 in particular supports phagocyte-mediated inflammation but can also skew type 1 into type 2 immune responses or enhance immunosuppressive activity of Treg and MDSC [55]. PGE2 has been one of the first effector molecules to be identified in MSC repertoire. Despite the pivotal role of iNOS in MSC therapeutic efficacy [50], Bouffi and colleagues demonstrated that IL-6 $6^{-/-}$MSC mimicked iNOS $^{-/-}$phenotype in the CIA model. IL- $6^{-/-}$MSC showed a concomitant down-regulation of the immunoregulatory molecule PGE2. The parallel down-modulation of IL-6 and PGE2 has recently been described as a result of the genetic impairment of the Notch signalling pathway in MSC from mice deficient for RBP-J, the transcription factor mediating Notch receptor signalling. RBP-J ${ }^{-/-}$MSC were unable, as compared to their wild type counterpart, to increase survival in a murine model of acute GvHD [56]. It has been also demonstrated that Notch-dependant up-regulation of COX-2 could be due to a self-activating process mediated by the in vivo aggregation of MSC into spheroids in a non-inflammatory context [57].

Although MSC immunosuppressive mechanisms mediated by amino acid depleting enzymes such as iNOS and IDO, or inhibition of COX-2 appear to be prominent in vivo, further interactions and cross-talk with other immuneregulatory cell types, cytokines and effector molecules have been proposed. MSC can reshape the inflammatory microenvironment modulating the expression of pro- and anti- inflammatory cytokines, such as IL-2, IFN- $\gamma /$ TGF- $\beta 1$, IL-10, as well as skewing Th1/Th2 responses and Treg/Th17 balance in a number of in vivo models comprising cardiac [58], renal [59] and skin [60] allograft rejection as well as different models of inflammatory colitis [61-63]. MSC can impair the immune response through down-modulation of MHCII and CD69 expression on CD11 ${ }^{+}$cells [64], or the inhibition of CCR7 and CD $49 \mathrm{~d} \beta 1$, counteracting DC migration to lymph nodes in acute GvHD $[65,66]$.

MSC showed renoprotective capacity in a model of ischemia reperfusion injury by inducing $\mathrm{CD} 11 \mathrm{c}^{+}$cells to secrete IL-10. In this model CD11 $\mathrm{c}^{+}$ cell depletion caused complete abrogation of the MSC renoprotective action, 
whilst the adoptive transfer of wild-type but not $\mathrm{IL}-10^{-/-} \mathrm{CD} 11 \mathrm{c}^{+}$cells restored the therapeutic activity [67]. MSC can ameliorate the clinical severity of ischaemic injury by modulating the infiltration of M2 macrophages in kidney [68] or heart [69]. MSC have been shown to mediate the expansion of human MDSC in vitro through hepatocyte growth factor (HGF) [70] and growth-regulated oncogene (GRO) chemokines [71]. In contrast, in vivo tumor-mediated generation of MDSC was inhibited by MSC in a model of murine hepatoma, ameliorating mouse survival [72].

\section{How can MSC Be Better Studied In Vivo?}

In-depth characterization of the immune-modulatory properties of MSC in vivo is impaired by the lack of appropriate tools to track and retrieve them from the recipient. Understanding where MSC home and engraft would give vital information on how the environment affects MSC immunosuppressive licensing and therapeutic targets. Furthermore, information about MSC biodistribution is likely to generate important pharmacokinetic data for the regulatory issues associated with clinical use [73].

Despite the existence of a number of strategies for cell tracking, not all of them are effective to visualise MSC after infusion. Tracers like 5-bromo2'-deoxyuridine (BrdU), GFP or luciferase, although extensively exploited, suffer, particularly when used for non-invasive imaging, of low sensitivity and non-specific signal due to protein re-uptake by recipient phagocytes [73].

Magnetic resonance imaging (MRI) could provide an interesting approach especially in applications that require scanning access to deep anatomical districts and organs. By way of an example, MSC transduced with ferritin heavy chain (FTH), which promote iron incorporation, have been used to track the infused cells without the transgene changing MSC biological properties [74]. On the other hand MSC labelled with the standard MRI contrast agent gadolinium via lipid transfection [75] or superparamagnetic iron oxide (SPIO) nanoparticles [76] can also be monitored without the necessity of a genetic manipulation of MSC. Nanoparticle-labelled MSC have been used in a mouse model of Chagas cardiomyopathy. MRI was used to demonstrate either the absence of MSC homing to the cardiac tissue (evidence that comes out in favour of indirect effects) and the reduction of hart dilatation in MSC-treated group [77]. ${ }^{111}$ Indium-oxine has been used to track MSC injected in patients with advanced cirrhosis, showing that most MSC were able to reach the liver and spleen within $48 \mathrm{~h}$ from infusion and still detectable 10 days later [78]. An alternative to MRI could be computed tomography (CT) with gold 
nanoparticles (GNP) as contrast agent. GNP-loaded human MSC have been found to home to rat brain and persist one month after infusion in FSL rats, a genetic model for depression [79].

Positron emission tomography (PET) has also been used for MSC non-invasive imaging. MSC have been successfully labelled and tracked with ${ }^{18}$ F-FDG, a positron-emitting glucose analogue that is taken up and metabolically trapped by cells, both in animal models [80,81] and in patient with acute myocardial infarction [82].

Although MSC tracking could help to explain the modalities of their therapeutic effects, a further crucial piece of information necessary to characterise their molecular activity in vivo is the possibility to recover the cells from the recipient after their administration. Embedding MSCs into engineered scaffolds can be a suitable option for both clinical applications and to address scientific questions. These materials can protect MSC from immunological rejection and increase their persistence in situ. Furthermore, they offer the opportunity to retrieve the cells after their implantation. Different types of polymeric scaffolds are available, including porous or nanofibrous matrices, sol-gel transition hydrogels and porous microspheres, in which cells can be embedded or seeded [83]. Alginate has been successfully used for MSC. It has been demonstrated that, after encapsulation, MSC retain their progenitor properties [84] and do not undergo cell proliferation [85]. Encapsulation does not impede in vitro MSC licensing by IFN- $\gamma$ and TNF- $\alpha$ treatment [86] and encapsulated MSC appear to retain in vivo clinical efficacy in a model of spinal cord trauma, in which they promote M2 differentiation of macrophages in the injured tissue [86]. In a model of acute GvHD, encapsulated MSC implanted subcutaneously have been demonstrated to be effective and superior to control MSC administered intravenously in improving clinical score and survival [87]. Similar results have been reported for the use of MSC in the treatment of liver fibrosis [88]. Human BM-MSC, cultured and delivered in a poly-lactic-co-glycolic acid nanofiber scaffold promoted the reduction of systemic inflammation in CIA [89]. In another study, MSC cultured on collagen sponges were transplanted into rat calvarial defects and efficiently recovered after 7 and 14 days from transplant for genomic profile characterization [90]. Lastly, MSC multi-layered cell sheet coupled with a porous acellular scaffold from bovine pericardium have been used to patch infarcted rat heart and demonstrated to produce improved ventricular function. Patches recovered after 12 weeks showed that a substantial portion of implanted MSC had differentiated into myofibroblast or smooth muscle cells [91]. 


\section{Concluding Remarks}

Despite the large number of in vitro studies, the mechanisms of MSC therapeutic activity in vivo remain unclear. Technical expedient aimed at retrieving MSC after their transplantation into recipients would offer the possibility to understand their function by interrogating MSC gene expression, proteomic and metabolomics profiles. Scaffolds can be an excellent tool to address this problem although permeability to host cells that is a function of pore size [92], topography, biochemical properties [93], changes in cell-adhesion and mechanotransduction (hydrodynamic and mechanical stress) [94] has to be taken into account in order to modulate and interpret correctly the contribution of soluble factors and cell-to-cell contact to MSC immunosuppressive licensing and effector mechanisms.

\section{References}

[1] A. J. Friedenstein, R. K. Chailakhjan, and K. S. Lalykina. "The development of fibroblast colonies in monolayer cultures of guineapig bone marrow and spleen cells." Cell Tissue Kinet 3, 393-403. (1970).

[2] E. Schipani and H. M. Kronenberg. "Adult mesenchymal stem cells," in StemBook, ed Cambridge (MA): Harvard Stem Cell Institute Copyright: (c) 2008 Ernestina Schipani and Henry M. Kronenberg. (2008).

[3] A. I. Caplan. "Adult mesenchymal stem cells for tissue engineering versus regenerative medicine." J. Cell Physiol. 213, 341-347. (2007).

[4] J. Kramer, F. Dazzi, M. Dominici, P. Schlenke, and W. Wagner. "Clinical perspectives of mesenchymal stem cells." Stem Cells Int. 2012, 684827. (2012).

[5] D. T. Shih and T. Burnouf. "Preparation, quality criteria, and properties of human blood platelet lysate supplements for ex vivo stem cell expansion." N. Biotechnol. 32, 199-211. (2015).

[6] W. E. Fibbe, F. Dazzi, and K. LeBlanc. "MSCs: science and trials." Nat. Med. 19, 812-813. (2013).

[7] E. Jones and R. Schäfer. "Biological differences between native and cultured mesenchymal stem cells: implications for therapies." Methods Mol. Biol. 1235, 105-120. (2015).

[8] M. F. Pittenger, A. M. Mackay, S. C. Beck, R. K. Jaiswal, R. Douglas, J. D. Mosca, et al. "Multilineage potential of adult human mesenchymal stem cells." Science 284, 143-147. (1999). 
[9] F. J. Lv, R. S. Tuan, K. M. Cheung, and V. Y. Leung. "Concise review: the surface markers and identity of human mesenchymal stem cells." Stem Cells 32, 1408-1419. (2014).

[10] M. Maleki, F. Ghanbarvand, M. Reza Behvarz, M. Ejtemaei, and E. Ghadirkhomi. "Comparison of mesenchymal stem cell markers in multiple human adult stem cells." Int. J. Stem Cells 7, 118-126. (2014).

[11] R. Hass, C. Kasper, S. Böhm, and R. Jacobs. "Different populations and sources of human mesenchymal stem cells (MSC): A comparison of adult and neonatal tissue-derived MSC." Cell Commun. Signal 9, 2. (2011).

[12] A. Shaer, N. Azarpira, M. H. Aghdaie, and E. Esfandiari. "Isolation and characterization of Human Mesenchymal Stromal Cells Derived from Placental Decidua Basalis; Umbilical cord Wharton's Jelly and Amniotic Membrane." Pak. J. Med. Sci. 30, 1022-1026. (2014).

[13] E. Collins, F. Gu, M. Qi, I. Molano, P. Ruiz, L. Sun, et al. "Differential efficacy of human mesenchymal stem cells based on source of origin," J. Immunol. 193, 4381-4390. (2014).

[14] W. Liu, F. Song, L. Ren, W. Guo, T. Wang, Y. Feng, et al. "The multiple functional roles of mesenchymal stem cells in participating in treating liver diseases." J. Cell Mol. Med. (2014).

[15] M. J. Goumans, J. A. Maring, and A. M. Smits. "A straightforward guide to the basic science behind cardiovascular cell-based therapies." Heart 100, 1153-1157. (2014).

[16] J. Bashir, A. Sherman, H. Lee, L. Kaplan, and J. M. Hare. "Mesenchymal stem cell therapies in the treatment of musculoskeletal diseases." $P M R$ 6, 61-69. (2014).

[17] M. Baghaban Eslaminejad and E. Malakooty Poor. "Mesenchymal stem cells as a potent cell source for articular cartilage regeneration," World J. Stem Cells 6, 344-354. (2014).

[18] B. Kristjánsson and S. Honsawek. "Current perspectives in mesenchymal stem cell therapies for osteoarthritis." Stem Cells Int. 2014, 194318. (2014).

[19] J. F. Swart and N. M. Wulffraat. "Mesenchymal stromal cells for treatment of arthritis." Best Pract. Res. Clin. Rheumatol. 28, 589-603. (2014).

[20] Y. Liu, J. Wu, Y. Zhu, and J. Han. "Therapeutic application of mesenchymal stem cells in bone and joint diseases," Clin. Exp. Med. 4, 13-24. (2014). 
[21] B. Skovrlj, G. Cunn, J. Guzman, and S. A. Qureshi. "Mesenchymal stem cell technology in the treatment of degenerative disc disease." J. Neurosurg. Sci. (2014).

[22] M. Boido, A. Piras, V. Valsecchi, G. Spigolon, K. Mareschi, I. Ferrero, et al. "Human mesenchymal stromal cell transplantation modulates neuroinflammatory milieu in a mouse model of amyotrophic lateral sclerosis." Cytotherapy 16, 1059-1072. (2014).

[23] L. Mazzini, K. Mareschi, I. Ferrero, M. Miglioretti, A. Stecco, S. Servo, et al. "Mesenchymal stromal cell transplantation in amyotrophic lateral sclerosis: a long-term safety study." Cytotherapy 14, 56-60. (2012).

[24] K. A. Chang, H. J. Kim, Y. Joo, S. Ha, and Y. H. Suh. "The therapeutic effects of human adipose-derived stem cells in Alzheimer's disease mouse models." Neurodegener. Dis. 13, 99-102. (2014).

[25] N. Kim and S. G. Cho. "Clinical applications of mesenchymal stem cells." Korean J. Int. Med. 28, 387-402. (2013).

[26] E. Buzhor, L. Leshansky, J. Blumenthal, H. Barash, D. Warshawsky, Y. Mazor, et al. "Cell-based therapy approaches: the hope for incurable diseases." Regen Med. 9, 649-672. (2014).

[27] K. Le Blanc, F. Frassoni, L. Ball, F. Locatelli, H. Roelofs, I. Lewis, et al. "Mesenchymal stem cells for treatment of steroid-resistant, severe, acute graft-versus-host disease: a phase II study." Lancet 371, 1579-1586. (2008).

[28] J. Tan, W. Wu, X. Xu, L. Liao, F. Zheng, S. Messinger, et al. "Induction therapy with autologous mesenchymal stem cells in living-related kidney transplants: a randomized controlled trial." JAMA 307, 1169-77. (2012).

[29] R. Ciccocioppo, M. E. Bernardo, A. Sgarella, R. Maccario, M. A. Avanzini, C. Ubezio, et al. "Autologous bone marrow-derived mesenchymal stromal cells in the treatment of fistulising Crohn's disease." Gut 60, 788-798. (2011).

[30] P. Connick, M. Kolappan, C. Crawley, D. J. Webber, R. Patani, A. W. Michell, et al. "Autologous mesenchymal stem cells for the treatment of secondary progressive multiple sclerosis: an open-label phase 2a proofof-concept study." Lancet Neurol. 11, 150-156. (2012).

[31] I. Müller, S. Lymperi, and F. Dazzi. "Mesenchymal stem cell therapy for degenerative inflammatory disorders." Curr. Opin. Organ. Transplant. 13, 639-644. (2008).

[32] D. Polchert, J. Sobinsky, G. Douglas, M. Kidd, A. Moadsiri, E. Reina, et al. "IFN-gamma activation of mesenchymal stem cells for treatment 
and prevention of graft versus host disease." Eur. J. Immunol. 38, 1745-1755. (2008).

[33] G. Ren, L. Zhang, X. Zhao, G. Xu, Y. Zhang, A. I. Roberts, et al. "Mesenchymal stem cell-mediated immunosuppression occurs via concerted action of chemokines and nitric oxide." Cell Stem Cell 2, 141-150. (2008).

[34] M. Krampera. "Mesenchymal stromal cell 'licensing': a multistep process." Leukemia 25, 1408-1414. (2011).

[35] L. Wang, Y. Zhao, and S. Shi. "Interplay between mesenchymal stem cells and lymphocytes: implications for immunotherapy and tissue regeneration." J. Dent. Res. 91, 1003-1010. (2012).

[36] I. Marigo and F. Dazzi. "The immunomodulatory properties of mesenchymal stem cells." Semin. Immunopathol. 33, 593-602. (2011).

[37] P. Renner, E. Eggenhofer, A. Rosenauer, F. C. Popp, J. F. Steinmann, P. Slowik, et al. "Mesenchymal stem cells require a sufficient, ongoing immune response to exert their immunosuppressive function." Transplant Proc. 41, 2607-2611. (2009).

[38] E. Valencic, C. Loganes, S. Cesana, E. Piscianz, G. Gaipa, E. Biagi, et al. "Inhibition of mesenchymal stromal cells by pre-activated lymphocytes and their culture media." Stem Cell Res. Ther. 5, 3. (2014).

[39] J. Su, X. Chen, Y. Huang, W. Li, J. Li, K. Cao, et al. "Phylogenetic distinction of iNOS and IDO function in mesenchymal stem cellmediated immunosuppression in mammalian species." Cell Death Differ. 21, 388-396. (2014).

[40] G. Ren, J. Su, L. Zhang, X. Zhao, W. Ling, A. L'huillie, et al. "Species variation in the mechanisms of mesenchymal stem cell-mediated immunosuppression." Stem Cells 27, 1954-1962. (2009).

[41] R. Meisel, S. Brockers, K. Heseler, O. Degistirici, H. Bülle, C. Woite, et al. "Human but not murine multipotent mesenchymal stromal cells exhibit broad-spectrum antimicrobial effector function mediated by indoleamine 2,3-dioxygenase." Leukemia 25, 648-654. (2011).

[42] K. Schroder, K. M. Irvine, M. S. Taylor, N. J. Bokil, K. A. Le Cao, K. A. Masterman, et al. "Conservation and divergence in Toll-like receptor 4-regulated gene expression in primary human versus mouse macrophages." Proc. Natl. Acad Sci. U.S.A. 109, E944-E953. (2012).

[43] M. Orciani, A. Campanati, E. Salvolini, G. Lucarini, G. Di Benedetto, A. Offidani, et al. "The mesenchymal stem cell profile in psoriasis." Br. J. Dermatol. 165, 585-592. (2011). 
[44] H. J. Ball, F. F. Jusof, S. M. Bakmiwewa, N. H. Hunt, and H. J. Yuasa. "Tryptophan-catabolizing enzymes - party of three." Front. Immunol. 5:485. (2014).

[45] K. English, F. P. Barry, C. P. Field-Corbett, and B. P. Mahon. "IFN-gamma and TNF-alpha differentially regulate immunomodulation by murine mesenchymal stem cells." Immunol. Lett. 110, 91-100. (2007).

[46] H. Y. Jui, C. H. Lin, W. T. Hsu, Y. R. Liu, R. B. Hsu, B. L. Chiang, et al. "Autologous mesenchymal stem cells prevent transplant arteriosclerosis by enhancing local expression of interleukin-10, interferon- $\gamma$, and indoleamine 2,3-dioxygenase." Cell Transplant 21, 971-984. (2012).

[47] W. Ge, J. Jiang, J. Arp, W. Liu, B. Garcia, and H. Wang. "Regulatory T-cell generation and kidney allograft tolerance induced by mesenchymal stem cells associated with indoleamine 2,3-dioxygenase expression." Transplantation 90, 1312-1320. (2010).

[48] D. Wang, X. Feng, L. Lu, J. E. Konkel, H. Zhang, Z. Chen, et al. "A CD8 $\mathrm{T}$ cell/indoleamine 2,3-dioxygenase axis is required for mesenchymal stem cell suppression of human systemic lupus erythematosus." Arthritis Rheumatol. 66, 2234-2245. (2014).

[49] D. Chabannes, M. Hill, E. Merieau, J. Rossignol, R. Brion, J. P. Soulillou, et al. "A role for heme oxygenase-1 in the immunosuppressive effect of adult rat and human mesenchymal stem cells." Blood 110, 3691-3694. (2007).

[50] C. Bouffi, C. Bony, G. Courties, C. Jorgensen, and D. Noël. "IL-6dependent PGE2 secretion by mesenchymal stem cells inhibits local inflammation in experimental arthritis," PLOS ONE 5:e14247. (2010).

[51] L. Zhang, R. J. Dang, H. Li, P. Li, Y. M. Yang, X. M. Guo, et al. "SOCS1 regulates the immune modulatory properties of mesenchymal stem cells by inhibiting nitric oxide production." PLOS ONE 9:e97256. (2014).

[52] Y. Huang, P. Yu, W. Li, G. Ren, A. I. Roberts, W. Cao, et al. "p53 regulates mesenchymal stem cell-mediated tumor suppression in a tumor microenvironment through immune modulation." Oncogene 33, 3830-3838. (2014).

[53] D. Claar, T. V. Hartert, and R. S. Peebles. "The role of prostaglandins in allergic lung inflammation and asthma." Expert Rev. Respir Med. 1-18. (2014).

[54] K. Kawahara, H. Hohjoh, T. Inazumi, S. Tsuchiya, and Y. Sugimoto. "Prostaglandin E2-induced inflammation: Relevance of prostaglandin E receptors." Biochim. Biophys. Acta (2014). 
[55] P. Kalinski. "Regulation of immune responses by prostaglandin E2." J. Immunol. 188, 21-28. (2012).

[56] Y. C. Wang, S. H. Wang, Y. N. Wei, D. W. Du, H. Xu, C. C. Gao, et al. "Notch-RBP-J signaling is required by bone marrow stromal cells for the treatment of acute graft versus host disease." Stem Cell Res. 11, 721-735. (2013).

[57] T. J. Bartosh, J. H. Ylöstalo, N. Bazhanov, J. Kuhlman, and D. J. Prockop. "Dynamic compaction of human mesenchymal stem/precursor cells into spheres self-activates caspase-dependent IL1 signaling to enhance secretion of modulators of inflammation and immunity (PGE2, TSG6, and STC1)." Stem Cells 31, 2443-2456. (2013).

[58] Y. Qiu, M. M. Yun, X. Han, R. Zhao, E. Zhou, and S. Yun. "Human umbilical cord mesenchymal stromal cells suppress MHC class II expression on rat vascular endothelium and prolong survival time of cardiac allograft." Int. J. Clin. Exp. Med. 7, 1760-1767. (2014).

[59] M. Koch, A. Lehnhardt, X. Hu, B. Brunswig-Spickenheier, M. Stolk, V. Bröcker, et al. "Isogeneic MSC application in a rat model of acute renal allograft rejection modulates immune response but does not prolong allograft survival." Transpl. Immunol. 29, 43-50. (2013).

[60] R. A. Larocca, P. M. Moraes-Vieira, E. J. Bassi, P. Semedo, D. C. de Almeida, M. B. da Silva, et al. "Adipose tissue-derived mesenchymal stem cells increase skin allograft survival and inhibit Th-17 immune response." PLOS ONE 8:e76396. (2013).

[61] M. A. González, E. Gonzalez-Rey, L. Rico, D. Büscher, and M. Delgado. "Adipose-derived mesenchymal stem cells alleviate experimental colitis by inhibiting inflammatory and autoimmune responses." Gastroenterology 136, 978-989. (2009).

[62] E. Gonzalez-Rey, P. Anderson, M. A. González, L. Rico, D. Büscher, and M. Delgado. "Human adult stem cells derived from adipose tissue protect against experimental colitis and sepsis." Gut 58, 929-939. (2009).

[63] Q. Q. Chen, L. Yan, C. Z. Wang, W. H. Wang, H. Shi, B. B. Su, et al. "Mesenchymal stem cells alleviate TNBS-induced colitis by modulating inflammatory and autoimmune responses." World J. Gastroenterol. 19, 4702-4717. (2013).

[64] H. Li, Z. Guo, H. Zhu, X. S. Li, X. Jiang, H. Yao, et al. "Transplanted mesenchymal stem cells can inhibit the three developmental stages of murine acute graft-versus-host disease." In Vivo 24, 659-666. (2010). 
[65] S. Chiesa, S. Morbelli, S. Morando, M. Massollo, C. Marini, A. Bertoni, et al. "Mesenchymal stem cells impair in vivo T-cell priming by dendritic cells." Proc. Natl. Acad Sci. U.S.A. 108, 17384-1739. (2011).

[66] H. Li, Z. Guo, X. Jiang, H. Zhu, X. Li, and N. Mao. "Mesenchymal stem cells alter migratory property of $\mathrm{T}$ and dendritic cells to delay the development of murine lethal acute graft-versus-host disease." Stem Cells 26, 2531-2541. (2008).

[67] M. G. Kim, S. H. Kim, H. Noh, Y. S. Ko, H. Y. Lee, S. K. Jo, et al. "CD11 $\mathrm{c}^{+}$cells partially mediate the renoprotective effect induced by bone marrow-derived mesenchymal stem cells." PLOS ONE 8:e72544. (2013).

[68] Y. Geng, L. Zhang, B. Fu, J. Zhang, Q. Hong, J. Hu, et al. "Mesenchymal stem cells ameliorate rhabdomyolysis-induced acute kidney injury via the activation of M2 macrophages." Stem Cell Res. Ther. 5, 80. (2014).

[69] D. I. Cho, M. R. Kim, H. Y. Jeong, H. C. Jeong, M. H. Jeong, S. H. Yoon, et al. "Mesenchymal stem cells reciprocally regulate the M1/M2 balance in mouse bone marrow-derived macrophages." Exp. Mol. Med. 46:e70. (2014).

[70] B. L. Yen, M. L. Yen, P. J. Hsu, K. J. Liu, C. J. Wang, C. H. Bai, et al. "Multipotent human mesenchymal stromal cells mediate expansion of myeloid-derived suppressor cells via hepatocyte growth factor/c-met and STAT3.” Stem Cell Rep. 1, 139-151. (2013).

[71] H. W. Chen, H. Y. Chen, L. T. Wang, F. H. Wang, L. W. Fang, H. Y. Lai, et al. "Mesenchymal stem cells tune the development of monocytederived dendritic cells toward a myeloid-derived suppressive phenotype through growth-regulated oncogene chemokines." J. Immunol. 190, 5065-5077. (2013).

[72] X. Su, L. Zhang, J. Ye, L. Yang, Y. Li, and Y. Wang. "Bone marrow mesenchymal stem cells suppress ascitogenous hepatoma progression in BALB/c mouse through reducing myeloid-derived suppressor cells." Biomed. Mater Eng. 25, 167-177. (2015).

[73] L. Sensebé and S. Fleury-Cappellesso. "Biodistribution of mesenchymal stem/stromal cells in a preclinical setting." Stem Cells Int. 2013, 678063. (2013).

[74] H. S. Kim, J. Woo, Y. Choi, E. H. Hwang, S. K. Choi, K. W. Cho, et al. "Noninvasive MRI and multilineage differentiation capability of ferritintransduced human mesenchymal stem cells." NMR Biomed (2014).

[75] K. Geng, Z. X. Yang, D. Huang, M. Yi, Y. Jia, G. Yan, et al. "Tracking of mesenchymal stem cells labeled with gadolinium diethylenetriamine 
pentaacetic acid by $7 \mathrm{~T}$ magnetic resonance imaging in a model of cerebral ischemia." Mol. Med. Rep. 11, 954-960. (2015).

[76] P. Hua, Y. Y. Wang, L. B. Liu, J. L. Liu, J. Y. Liu, Y. Q. Yang, et al. "In vivo magnetic resonance imaging tracking of transplanted superparamagnetic iron oxidelabeled bone marrow mesenchymal stem cells in rats with myocardial infarction." Mol. Med. Rep. 11, 113-1120. (2015).

[77] Jasmin, L. A. Jelicks, H. B. Tanowitz, V. M. Peters, R. Mendez-Otero, A. C. Campos de Carvalho, et al. "Molecular imaging, biodistribution and efficacy of mesenchymal bone marrow cell therapy in a mouse model of Chagas disease." Microbes Infect 16, 923-935. (2014).

[78] A. Gholamrezanezhad, S. Mirpour, M. Bagheri, M. Mohamadnejad, K. Alimoghaddam, L. Abdolahzadeh, et al. "In vivo tracking of 111Inoxine labeled mesenchymal stem cells following infusion in patients with advanced cirrhosis." Nucl. Med. Biol. 38, 961-917. (2011).

[79] O. Betzer, A. Shwartz, M. Motiei, G. Kazimirsky, I. Gispan, E. Damti, et al. "Nanoparticle-based CT imaging technique for longitudinal and quantitative stem cell tracking within the brain: application in neuropsychiatric disorders." ACS Nano 8, 9274-9285. (2014).

[80] E. Wolfs, T. Struys, T. Notelaers, S. J. Roberts, A. Sohni, G. Bormans, et al. "18F-FDG labeling of mesenchymal stem cells and multipotent adult progenitor cells for PET imaging: effects on ultrastructure and differentiation capacity." J. Nucl. Med. 54, 447-454. (2013).

[81] M. Kantarcioglu, B. Caliskan, H. Demirci, O. Karacalioglu, M. Kekilli, Z. Polat, et al. "The efficacy of mesenchymal stem cell transplantation in caustic esophagus injury: an experimental study." Stem Cells Int. 2014, 939674. (2014).

[82] M. Hofmann, K. C. Wollert, G. P. Meyer, A. Menke, L. Arseniev, B. Hertenstein, et al. "Monitoring of bone marrow cell homing into the infarcted human myocardium." Circulation 111, 2198-2202. (2005).

[83] T. Garg, O. Singh, S. Arora, and R. Murthy. "Scaffold: a novel carrier for cell and drug delivery." Crit. Rev. Ther. Drug Carrier Syst. 29, 1-63. (2012).

[84] A. Goren, N. Dahan, E. Goren, L. Baruch, and M. Machluf. "Encapsulated human mesenchymal stem cells: a unique hypoimmunogenic platform for long-term cellular therapy." FASEB J. 24, 22-31. (2010).

[85] J. F. Markusen, C. Mason, D. A. Hull, M. A. Town, A. B. Tabor, M. Clements, et al. "Behavior of adult human mesenchymal stem cells entrapped in alginate-GRGDY beads." Tissue Eng. 12, 821-830. (2006). 
[86] J. Barminko, J. H. Kim, S. Otsuka, A. Gray, R. Schloss, M. Grumet, et al. "Encapsulated mesenchymal stromal cells for in vivo transplantation." Biotechnol. Bioeng. 108, 2747-2758. (2011).

[87] L. Zanotti, A. Sarukhan, E. Dander, M. Castor, J. Cibella, C. Soldani, et al. "Encapsulated mesenchymal stem cells for in vivo immunomodulation." Leukemia 27, 500-503. (2013).

[88] R. P. Meier, R. Mahou, P. Morel, J. Meyer, E. Montanari, Y. D. Muller, et al. "Microencapsulated human mesenchymal stem cells decrease liver fibrosis in mice." J. Hepatol. (2014).

[89] X. Zhang, K. Yamaoka, K. Sonomoto, H. Kaneko, M. Satake, Y. Yamamoto, et al. "Local delivery of mesenchymal stem cells with poly-lactic-co-glycolic Acid nano-fiber scaffold suppress arthritis in rats." PLoS ONE 9:e114621. (2014).

[90] S. J. Hwang, T. H. Cho, and I. S. Kim. "In vivo gene activity of human mesenchymal stem cells after scaffold-mediated local transplantation." Tissue Eng Part A 20, 2350-2364. (2014).

[91] C. H. Chen, H. J. Wei, W. W. Lin, I. Chiu, S. M. Hwang, C. C. Wang, et al. "Porous tissue grafts sandwiched with multilayered mesenchymal stromal cell sheets induce tissue regeneration for cardiac repair." Cardiovasc Res. 80, 88-95. (2008).

[92] J. H. Brauker, V. E. Carr-Brendel, L. A. Martinson, J. Crudele, W. D. Johnston, and R. C. Johnson. "Neovascularization of synthetic membranes directed by membrane microarchitecture." J. Biomed. Mater Res. 29, 1517-1524. (1995).

[93] T. A. Telemeco, C. Ayres, G. L. Bowlin, G. E. Wnek, E. D. Boland, N. Cohen, et al. "Regulation of cellular infiltration into tissue engineering scaffolds composed of submicron diameter fibrils produced by electrospinning." Acta Biomater. 1, 377-385. (2005).

[94] B. J. Lawrence and S. V. Madihally. "Cell colonization in degradable 3D porous matrices.” Cell Adh. Migr. 2, 9-16. (2008). 


\section{Biographies}

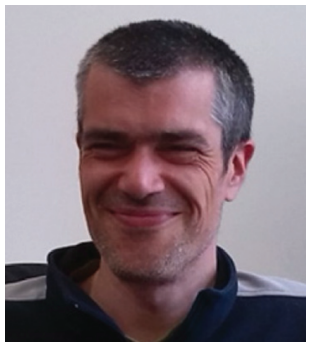

L. Dolcetti studied biology and got his $\mathrm{PhD}$ in oncology at the University of Padua. During the $\mathrm{PhD}$, he mainly worked on the biology of myeloid derived suppressor cells (MDSC), their phenotype and mechanisms of immunosuppression in the tumor microenvironment. In 2013 he moved to King's College London where he is currently studying the immuno-modulatory properties of mesenchymal stem/stromal cells.

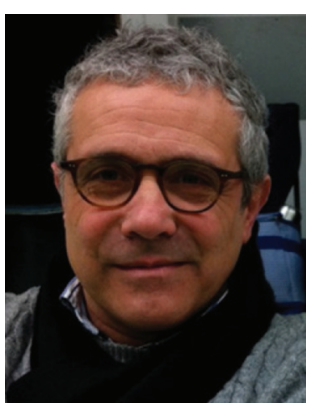

F. Dazzi got his medical degree and specialization in oncology at the University of Padua, the specialization in haematology at the University of Verona. He held the position of Professor of Stem Cell Biology and Honorary Consultant Haematologist at Imperial College London. He is currently Professor of Regenerative Medicine and Haematology, KHP Lead for Cellular Therapies and Consultant Haematologist at King's College London. 
